\title{
Discrete Patterns of Cross-Hemispheric Functional Connectivity Underlie Impairments of Spatial Cognition after Stroke
}

\author{
Radek Ptak, ${ }^{1,2,3}$ Alexia Bourgeois, ${ }^{1}$ Silvia Cavelti, ${ }^{1}$ Naz Doganci, ${ }^{1}$ Armin Schnider,,${ }^{1,2}$ and \\ Giannina Rita Iannotti ${ }^{1,4}$ \\ ${ }^{1}$ Laboratory of Cognitive Neurorehabilitation, Department of Clinical Neurosciences, Faculty of Medicine, University of Geneva, Geneva, 1206, \\ Switzerland, ${ }^{2}$ Division of Neurorehabilitation, University Hospitals of Geneva, Geneva, 1206, Switzerland, ${ }^{3}$ Faculty of Psychology and Educational \\ Sciences, University of Geneva, Geneva, 1205, Switzerland, and ${ }^{4}$ Swiss Foundation for Innovation and Training in Surgery, University Hospitals of \\ Geneva, Geneva, 1206, Switzerland
}

Despite intense research, the neural correlates of stroke-induced deficits of spatial cognition remain controversial. For example, several cortical regions and white-matter tracts have been designated as possible anatomic predictors of spatial neglect. However, many studies focused on local anatomy, an approach that does not harmonize with the notion that brain-behavior relationships are flexible and may involve interactions among distant regions. We studied in humans of either sex restingstate fMRI connectivity associated with performance in line bisection, reading and visual search, tasks commonly used for he clinical diagnosis of neglect. We defined left and right frontal, parietal, and temporal areas as seeds (or regions of interest, ROIs), and measured whole-brain seed-based functional connectivity (FC) and ROI-to-ROI connectivity in subacute right-hemisphere stroke patients. Performance on the line bisection task was associated with decreased FC between the right fusiform gyrus and left superior occipital cortex. Complementary increases and decreases of connectivity between both temporal and occipital lobes predicted reading errors. In addition, visual search deficits were associated with modifications of FC between left and right inferior parietal lobes and right insular cortex. These distinct connectivity patterns were substantiated by analyses of FC between left- and right-hemispheric ROIs, which revealed that decreased interhemispheric and right intrahemispheric FC was associated with higher levels of impairment. Together, these findings indicate that intrahemispheric and interhemispheric cooperation between brain regions lying outside the damaged area contributes to spatial deficits in a way that depends on the different cognitive components recruited during reading, spatial judgments, and visual exploration.

Key words: attention network; functional connectivity; parietal lobe; spatial cognition; spatial neglect; visual attention

\section{Significance Statement}

Focal damage to the right cerebral hemisphere may result in a variety of deficits, often affecting the domain of spatial cognition. The neural correlates of these disorders have traditionally been studied with lesion-symptom mapping, but this method fails to capture the network dynamics that underlie cognitive performance. We studied functional connectivity in patients with right-hemisphere stroke and found a pattern of correlations between the left and right temporo-occipital, inferior parietal, and right insular cortex that were distinctively predictive of deficits in reading, spatial judgment, and visual exploration. This finding reveals the importance of interhemispheric interactions and network adaptations for the manifestation of spatial deficits after damage to the right hemisphere.

Received Mar. 10, 2020; revised June 6, 2020; accepted July 4, 2020.

Author contributions: R.P. designed research; R.P. performed research; R.P. and G.R.I. analyzed data; R.P. wrote the first draft of the paper; R.P., A.B., S.C., N.D., A.S., and G.R.I. edited the paper; R.P., A.B., S.C., N.D., A.S., and G.R.I. wrote the paper.

R.P. was supported by Swiss National Science Foundation Grant 32003B-184702 and the Novartis Foundation for Medical-Biological Research Grant 16C183. A.S. was supported by Swiss National Science Foundation Grant 320030175472. Technical support was provided by staff from the Center for Biomedical Imaging (CIBM) of the Universities of Geneva and Lausanne, the EPFL and the University Hospitals of Geneva and Lausanne.

The authors declare no competing financial interests.

Correspondence should be addressed to Radek Ptak at radek.ptak@hcuge.ch.

https://doi.org/10.1523/JNEUROSCI.0625-20.2020

Copyright $\odot 2020$ the authors

\section{Introduction}

Traditionally, the study of spatial impairments after focal brain injury has focused heavily on local cortical anatomy (Vallar and Perani, 1986; Doricchi and Tomaiuolo, 2003; Mort et al., 2003; Karnath et al., 2004; Committeri et al., 2007; Golay et al., 2008; Medina et al., 2009). Only recently has this focus shifted toward possible structural disconnections underlying clinical syndromes, such as spatial neglect (Urbanski et al., 2011; Thiebaut de Schotten et al., 2014). Voxel-based lesion-symptom mapping (VLSM) and diffusion tensor imaging (DTI) have led to a complex picture, suggesting that numerous regions and fiber tracts 
contribute to such deficits (Chechlacz et al., 2012; Molenberghs et al., 2012; Pedrazzini and Ptak, 2020). This could reflect the observation that patients, though sharing an underlying "core" component, may differ on dimensions, such as egocentric/ allocentric, personal/extrapersonal, or near/far space neglect (Corbetta and Shulman, 2011; Sperber and Karnath, 2018). Alternatively, different inclusion criteria or issues of statistical power in an area of study that strongly relies on familywise error (FWE) control may have contributed to these intricate findings.

Despite decades of research, the nature of the association between brain anatomy and cognitive function remains difficult to specify, even more so as it may differ across brain areas and the class of function under investigation (Price and Friston, 2002). Functional imaging has led to the dismissal of the idea that this association is rigid (as in a one-to-one relationship), in favor of a more flexible connection (one-to-many, many-toone), or even the hypothesis that anatomic correlates of a function vary across situations according to current computational requirements (many-to-many) (Shallice and Cooper, 2011; Cole et al., 2013; Poldrack and Farah, 2015; Ptak et al., 2017). The latter perspective describes a brain that is highly dynamic and adaptable, which contrasts with the relatively static picture of brain function provided by lesion overlap methods.

Measures of resting-state functional connectivity (FC) provide the possibility to study distributed networks and their alteration as predictors of behavioral deficits after stroke in a complementary way to VLSM and DTI methods (Buckner et al., 2013; Bassett and Sporns, 2017). Connectome studies show that the human brain is intrinsically organized into large-scale networks (Yeo et al., 2011; Petersen and Sporns, 2015), which, when damaged, may give rise to widespread patterns of functional disconnection (Alstott et al., 2009; Buckner et al., 2009; Gratton et al., 2012). FC-behavior relationships have been revealed for deficits of spatial cognition, where some studies reported that interhemispheric FC between dorsal frontoparietal regions predicts deficits of lateralized attention after hemispheric stroke (He et al., 2007; Carter et al., 2010). Likewise, intrahemispheric frontoparietal disconnection was found to be associated with impairments of goal-directed attention (Fellrath et al., 2016). Interactions between functional networks within and across hemispheres appear to be particularly affected in patients with spatial neglect, suggesting that changes of functional networks may provide a specific signature of this disorder (Baldassarre et al., 2014).

Behavioral studies with brain-damaged patients regularly report dissociations of performance in different tests of spatial attention, a finding reflecting the involvement of discrete cognitive mechanisms. For example, damage to posterior and inferior parietal or premotor cortex differently affects cancellation and line bisection (Pedrazzini et al., 2017; Toba et al., 2017), personal and extrapersonal space (Committeri et al., 2007; Baas et al., 2011), orienting of attention (Molenberghs et al., 2008; Vandenberghe et al., 2012; Pedrazzini and Ptak, 2019), and reading (Lee et al., 2009; Medina et al., 2009). Here, we examined whether such distinct patterns of performance in tests of spatial cognition are predictable from resting-state FC. We assessed cancellation, line bisection, and reading performance in patients with right hemisphere stroke and established correlations with brainwide FC. Our findings identify distinct intrahemispheric and interhemispheric connectivity patterns as predictors of stroke-induced deficits of spatial cognition.

\section{Materials and Methods}

Participants. We recruited 30 patients (11 women) with first-ever right-hemisphere stroke while they were hospitalized at the Division of Neurorehabilitation, University Hospitals Geneva. Approval for this study was obtained from the local ethical committee (Geneva, Switzerland), and all participants gave written informed consent before being enrolled. Exclusion criteria were as follows: (1) presence of prior strokes or multiple lacunar lesions; (2) inability to participate to MRI (e.g., presence of metallic parts in the body); (3) severe cognitive impairment precluding administration or interpretation of clinical tests (e.g., severe hypovigilance, confusion, or dementia); and (4) presence of other neurologic or psychiatric diseases (e.g., major depression). We did not select participants based on their performance in any of the clinical tests.

Mean age of patients was $63 \pm 13.1$ years, and the mean delay of testing after stroke onset was $42.6 \mathrm{~d}$. All participants had a standard neurologic examination, including visual field testing through digital confrontation and/or a campimetry test (detection of running numbers on a 24 inch screen).

Experimental procedures and stimuli. They were administered the following clinical tests: line bisection (Ronchi et al., 2012), symbol cancellation (the Bells test) (Gauthier et al., 1989), letter cancellation (Ptak et al., 2007), and word reading (Ptak et al., 2012). The reading test consisted of 40 compound words presented in 5 columns on an A4 sheet of paper. This test has previously been used to identify patients with spatial dyslexia, a reading disorder associated with left neglect that is characterized by omissions and substitutions of word beginnings.

Performance in these tests may be taken as cutoff criterion to identify clinical groups for group-based FC analyses, or may be entered as continuous measure in regression-based FC analyses. In this study, we performed both types of analyses and therefore defined groups showing clinically relevant neglect in line bisection, symbol cancellation, or reading tests based on cutoff criteria from previous normative studies. For the reading test, we first applied the criteria of Ellis et al. (1987) to classify reading errors as omissions, substitutions, additions, or mixed errors. Patients were then classified as belonging to a spatial dyslexia group if they reached the cutoff of $n \geq 4$ lateralized reading errors. Seven patients were identified as spatial dyslexics using this criterion. They missed on average 13.7 ( \pm 9.5 ) words completely and transformed 7.2 ( \pm 5.5 ) words compared with $2.6( \pm 3$ ) words missed and $0.3( \pm 0.6)$ transformations by the nondyslexia group. Neglect on line bisection was defined by using the cutoff of $\geq 2$ SDs of healthy control performance. This criterion identified 9 patients, who had an average bisection error of $9 \%( \pm 5.1)$ compared with $1.4 \%( \pm 1.6)$ of the non-neglect group. Finally, the cutoff score used to define left neglect on the cancellation test was $\geq 3$ omissions on the left side of the sheet. This criterion identified 13 patients, who missed on average 10.6 ( \pm 3.6) items on the left, compared with $1( \pm 1.3)$ item for the non-neglect group. Table 1 provides clinical characteristics and individual test results of all patients.

Experimental design and statistical analysis. The study focused on 30 human stroke patients of both sexes who to different degrees expressed deficits in simple tasks probing spatial cognition. We used clinical tests that evaluate elementary visuospatial performance and in which performance of healthy participants score at ceiling (e.g., a cutoff of two for the difference between left and right omissions is considered as pathologic performance on the Bells test) (Azouvi et al., 2002), precluding any correlational analysis with measures of connectivity. We therefore renounced testing controls and instead compared global patterns of connectivity in the patient group with anatomic and resting state (rs)-fMRI data of middle-aged healthy subjects from a previous study (Goveas et al., 2013) (data freely available through the 1000 Functional Connectomes Project at http://fcon_1000.projects.nitrc.org). We selected from this dataset the 27 oldest participants (12 women). The mean age of the control group was 55.9 ( \pm 6.1 ) years.

Group comparisons of ROI-to-ROI FC data were performed with ANOVA. Group comparisons of seed-based data applied independentsamples $t$ tests with a significance level $p<0.001$ and FWE-adjusted cluster-size correction.

MRI acquisition. Structural and functional MRI scans were acquired on a $3 \mathrm{~T}$ Trio scanner (Siemens Medical Solutions) in a single session, 
Table 1. Clinical characteristics and test results of the patient sample (inf./sup. quad.: inferior/superior quadrant)

\begin{tabular}{|c|c|c|c|c|c|c|c|c|}
\hline No. & Age (yr) & Time post stroke (days) & Visual field & Bells left omissions (of 15) & Letters left omissions (of 27) & Bisection (\% bias) & Reading errors & Spatial dyslexia errors \\
\hline 1 & 61.8 & 53 & Intact & 15 & 0 & -0.05 & 0 & 0 \\
\hline 2 & 78.9 & 13 & Hemianopia & 0 & 15 & 5.14 & 23 & 4 \\
\hline 3 & 69.0 & 48 & Intact & 0 & 0 & 0 & 0 & 0 \\
\hline 4 & 78.5 & 65 & Intact & 6 & 20 & 3.18 & 11 & 8 \\
\hline 5 & 62.6 & 56 & Intact & 1 & 2 & 0 & 0 & 0 \\
\hline 6 & 66.0 & 38 & Intact & 11 & 3 & 11.31 & 4 & 1 \\
\hline 7 & 71.7 & 39 & Hemianopia & 15 & 27 & 21.41 & 6 & 4 \\
\hline 8 & 66.2 & 30 & Intact & 1 & 0 & -0.94 & 1 & 0 \\
\hline 9 & 81.4 & 35 & Intact & 0 & 5 & 2.89 & 2 & 1 \\
\hline 10 & 66.6 & 50 & Hemianopia & 15 & 11 & 8.1 & 33 & 0 \\
\hline 11 & 33.6 & 21 & Intact & 0 & 0 & 0 & 0 & 19 \\
\hline 12 & 79.3 & 21 & Intact & 8 & 11 & -0.72 & 3 & 0 \\
\hline 13 & 51.8 & 43 & Intact & 0 & 2 & 2.82 & 1 & 0 \\
\hline 14 & 42.1 & 91 & Intact & 7 & 4 & 2.45 & 4 & 2 \\
\hline 15 & 69.5 & 48 & Inferior quadrant & 10 & 21 & 5.79 & 38 & 4 \\
\hline 16 & 65.6 & 52 & Intact & 0 & - & 7.11 & 1 & 0 \\
\hline 17 & 46.3 & 23 & Intact & 0 & 6 & 2.24 & 9 & 0 \\
\hline 18 & 62.2 & 37 & Intact & 7 & 6 & 3.96 & 6 & 0 \\
\hline 19 & 58.7 & 54 & Intact & 3 & 3 & 0.4 & 1 & 0 \\
\hline 20 & 59.4 & 73 & Hemianopia & 10 & 9 & 2.78 & 3 & 0 \\
\hline 21 & 70.3 & 37 & Intact & 15 & 27 & 8.58 & 15 & 4 \\
\hline 22 & 76.4 & 26 & Hemianopia & 3 & 3 & 4.9 & 24 & 0 \\
\hline 23 & 65.3 & 33 & Hemianopia & 13 & 12 & 8.22 & 6 & 1 \\
\hline 24 & 78.7 & 23 & Intact & 6 & 9 & 0.94 & 11 & 0 \\
\hline 25 & 55.9 & 55 & Intact & 2 & 3 & 0.78 & 2 & 1 \\
\hline 26 & 63.6 & 50 & Superior quadrant & 3 & 5 & 2.44 & 4 & 0 \\
\hline 27 & 28.3 & 18 & Intact & 2 & 0 & 2.73 & 5 & 1 \\
\hline 28 & 72.2 & 45 & Intact & 0 & 1 & 3.53 & 0 & 0 \\
\hline 29 & 77.6 & 45 & Intact & 3 & 1 & -0.57 & 5 & 4 \\
\hline 30 & 48.0 & 33 & Intact & 0 & 0 & 1.32 & 0 & 0 \\
\hline Mean & 63.6 & 41.8 & & 5.2 & 7.1 & 3.69 & 7.3 & 1.8 \\
\hline
\end{tabular}

which took place within a week from behavioral testing. A high-resolution (voxel size: $1 \mathrm{~mm}^{3}$ ) T1-weighted MPRAGE sequence (TR: $2300 \mathrm{~ms}$; TE: $1.94 \mathrm{~ms}$; flip angle $9^{\circ}$ ) and a T2-weighted (TR: $3200 \mathrm{~ms}$; TE: $407 \mathrm{~ms}$; flip angle $120^{\circ}$ ) structural scan were acquired for normalization and lesion delineation. The rs-fMRI scan consisted of 320 volumes acquired using T2*-weighted GRE EPI sequence (TR: $1500 \mathrm{~ms}$; TE: $35 \mathrm{~ms}$; voxel size $3 \times 3 \times 5 \mathrm{~mm}$; flip angle $85^{\circ}$ ). During acquisition, each subject's head was fixed with cushions to prevent motion, and participants lay in the dark, eyes closed. Control data were acquired on a 3T Signa GE scanner (GE Healthcare Technologies) and consisted of a high-resolution (voxel size: $1 \mathrm{~mm}^{3}$ ) 3D spoiled gradient-recalled echo anatomic scan (TR: $10 \mathrm{~ms}$; TE: $4 \mathrm{~ms}$; flip angle $12^{\circ}$ ) and 175 volume EPI sequence (TR: $2000 \mathrm{~ms}$; TE: $25 \mathrm{~ms}$; voxel size $3.75 \times 3.75 \times 4 \mathrm{~mm}$; flip angle $90^{\circ}$ ).

Lesion delineation and preprocessing of structural and functional MRI data. For each patient, a volumetric mask was created by delineating every lesion manually on the T2-weighted MRI scan in MRIcron software (Rorden et al., 2007). The structural T2 scan and the lesion mask were realigned and coregistered with the structural T1 using SPM12 (www.fil.ion.ucl.ac.uk/spm). Structural T1 scans were then segmented and normalized to the MNI (http://www.bic.mni.mcgill.ca) template space. These steps were performed with the Clinical Toolbox (Rorden et al., 2012), which offers an age-specific MRI template and automatically applies an enantiomorphic lesion mask to minimize normalization artifacts induced by abnormal tissue (Nachev et al., 2008). Normalization produced images of $2 \times 2 \times 2 \mathrm{~mm}$ voxel size.

Analysis of rs-fMRI data. We used the CONN toolbox (WhitfieldGabrieli and Nieto-Castanon, 2012) to normalize patients' functional volumes and for the complete preprocessing of control's structural and functional images (including segmentation of the structural T1 and normalization to MNI). Normalized images were smoothed with an $8 \mathrm{~mm}$ FWHM function. ART-based scrubbing was used to identify outlier volumes (Power et al., 2012). We corrected nuisance (physiological and movement artifacts) with a linear regression model, by inserting the following as confounds: (1) the six rigid-body parameters estimated from realignment, (2) averaged BOLD signals of white matter and CSF, and (3) the scrubbing parameters. Moreover, data were filtered with a bandpass of $0.008-0.09 \mathrm{~Hz}$ to remove low-frequency drift and high-frequency noise. Scrubbing identified on average $0.25 \%$ outlier scans in controls, and maximum framewise displacement was $<0.5 \mathrm{~mm}$ in all subjects. Two patients had an excessive number of outlier scans $(>13 \%)$ and were therefore eliminated from further analysis. The remaining 28 patients had on average $1.82 \%$ outlier scans identified by scrubbing and a maximal framewise displacement $<0.5 \mathrm{~mm}$.

We next defined seven cortical seeds (or ROIs) based on the AAL atlas (Tzourio-Mazoyer et al., 2002). We focused on regions that lie in the ventral and dorsal stream and are either central nodes in attention networks (angular gyrus [Ang]; supramarginal gyrus [SMG]; superior parietal lobule [PSup]; superior frontal cortex [FSup]; lateral inferior frontal cortex [FInf]) or have previously been identified as important predictors of spatial dyslexia (fusiform gyrus [Fus]; superior temporal gyrus [STG]) (Lee et al., 2009; Ptak et al., 2012). For seed-based FC analyses, we computed Fisher-transformed correlation and applied a threshold of $p<0.001$ (uncorrected) at the voxel level. The significance level for clusters was set to $p<0.05$, with an FWE correction for cluster volume (testing performed with $n=1000$ permutations). In all analyses, we examined seed-based FC of right-hemispheric ROIs and of homotopic regions of the left hemisphere. In a second step, we also computed ROIto-ROI connectivity between ipsilateral and contralateral ROIs. While we focused on cortical ROIs, seed-based FC was always computed with the rest of the brain, including subcortical regions, such as the thalamus; any involvement of the latter would therefore be identified in our analyses.

A major problem when examining FC in brain-injured patients is that the degree of connectivity in the injured area is unknown, as the damage seen on MRI is at the best a modest predictor of residual activity and areas with altered neurovascular coupling cannot easily be 


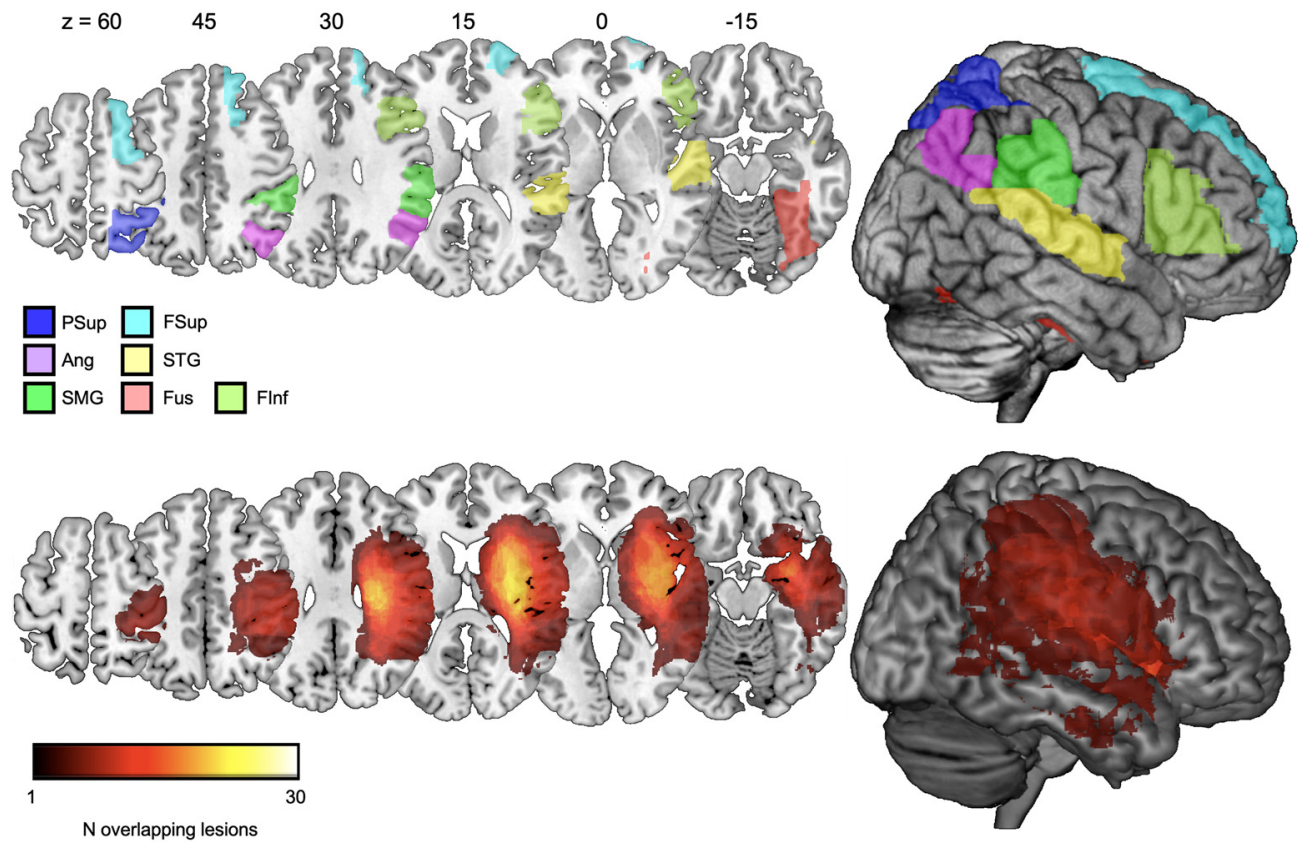

Figure 1. Top, Axial slices of the MNI-template brain showing the five ROls entered as seeds in FC analyses ( $z$ values show MNI coordinates). Bottom, Rendering of brain lesions of all participants. Color scale represents the number of patients with damage at each specific voxel.

identified. Stroke may affect the BOLD signal outside the damaged area and even in the intact hemisphere (Pineiro et al., 2002; Amemiya et al., 2012), and brain regions that are close to, but do not overlap with, damaged tissue may exhibit significant changes of connectivity ( $\mathrm{Lv}$ et al., 2013; Siegel et al., 2017). More importantly, areas with decreased BOLD responses may exhibit significant neural activity as measured with evoked potential methods (Rossini et al., 2004). Because of the insecurities regarding changes of neurovascular coupling in our patients, we decided to include injured voxels in the FC analyses. We quantified the relative damage to each ROI as well as the total lesion volume and examined their impact on FC by including them as covariates in the connectivity analyses.

\section{Results}

Lesion distribution and overlap with ROIs

Figure 1 shows the distribution of lesions in the patient group. Most patients shared damage in periventricular white matter, whereas cortical lesions were comparatively less frequent. The average overlap of each patient's lesion with each ROI was, respectively, as follows: 5.7\% (Fus), 7.2\% (Ang), 15.4\% (SMG), $2.1 \%$ (PSup), $15.4 \%$ (STG), $0.03 \%$ (FSup) and $10.2 \%$ (FInf). Thus, there was a modest involvement of damaged tissue in seed-ROIs used in the FC analyses.

\section{Global patterns of connectivity}

Before examining specific FC predictors of behavioral performance, we assessed global patterns of seed-based connectivity for both groups of participants. These analyses applied one-sample $t$ tests $(p<0.001$ and FWE cluster-size correction), examining whether connectivity at each voxel was significantly different from zero. In order to ensure that these analyses were based on a comparable number of functional images, we included only the first 175 volumes of the patient data. Figure 2 shows significant voxels identified in these seed-based analyses.

Analyses of control data revealed bilateral and largely symmetrical functional networks that were specific for each seedROI (Fig. 2). These networks implicated the following: (1) inferior and superior temporal cortex, lateral and superior occipital cortex, as well as the precentral and postcentral gyrus for the Fus seed; (2) inferior parietal, mid-temporal, premotor, dorsal prefrontal, frontopolar, and retrosplenial cortex for the Ang seed; (3) inferior and superior parietal cortex, STG, lateral PFC, and insula for the SMG seed; (4) superior and inferior parietal, dorsal premotor, lateral prefrontal, and lateral occipital cortex for the PSup seed; (5) the superior and middle temporal and lateral occipital cortex, SMG, insula, as well as the precentral and postcentral gyrus for the STG seed; (6) dorsal and ventral PFC, Ang, and middle temporal gyrus for the FSup seed; and (7) lateral PFC, SMG, medial, and STG for the FInf seed. As Figure 2 shows, there were only few negative correlations, mainly contralateral and with the SMG and Ang seeds. In strong contrast to controls, stroke patients exhibited FC with regions lying in the vicinity of the seed-ROI, whereas contralateral connectivity was limited to regions that were homologous with the seed.

These analyses identified the global patterns of connectivity but did not provide a direct comparison of absolute connectivity values across the two groups. We therefore ran a further analysis, which compared FC of both groups at the voxel level with an independent-samples $t$ test. The results revealed a pattern of significant differences that closely matched the general connectivity pattern of the control group (Figs. 2, left, 3), showing that controls had higher connectivity than stroke patients in cortical networks ipsilateral and contralateral to all right-hemisphere seed regions under focus.

We next performed ROI-to-ROI analyses by extracting correlations between right- and left-hemispheric ROI pairs and compared them across groups. This analysis was motivated by previous findings showing that focal damage after stroke is associated with a significant decrease of FC between homotopic regions (Siegel et al., 2016; Tang et al., 2016). We computed average connectivity values for each combination of the five rightand left-hemispheric ROIs and classified them according to one of four conditions: interhemispheric homotopic, interhemispheric heterotopic, left intrahemispheric, and right intrahemi- 
A

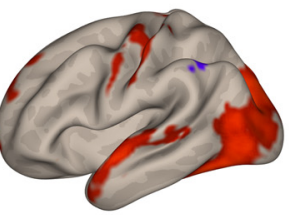

B

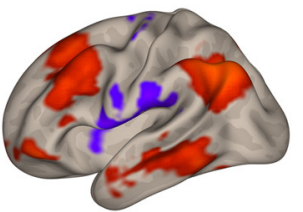

C

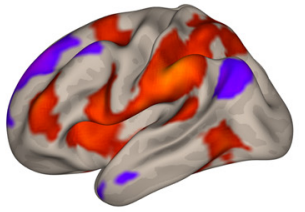

D

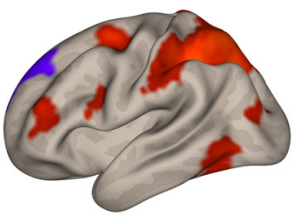

E

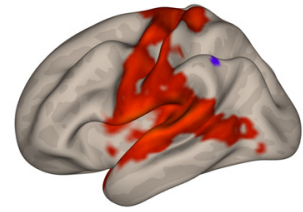

$\mathbf{F}$

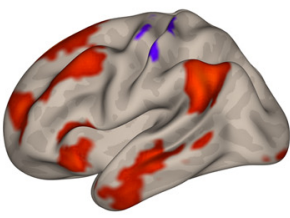

G

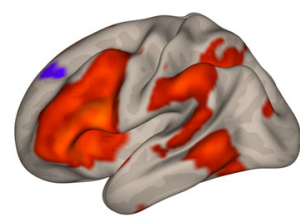

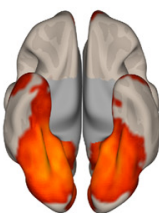
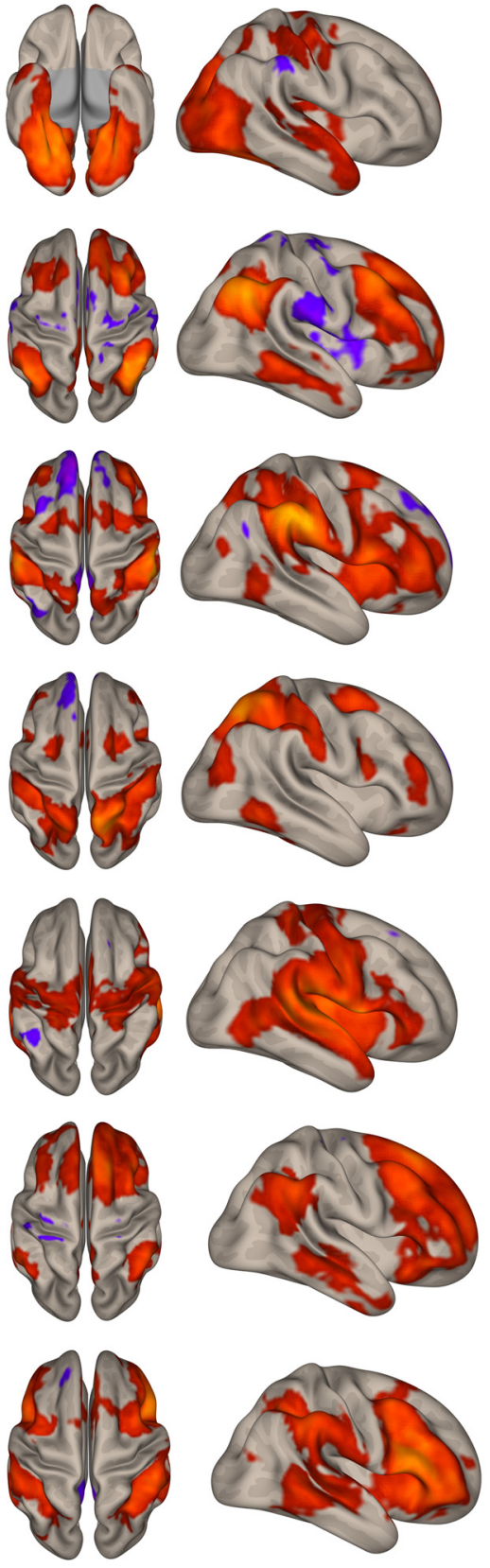
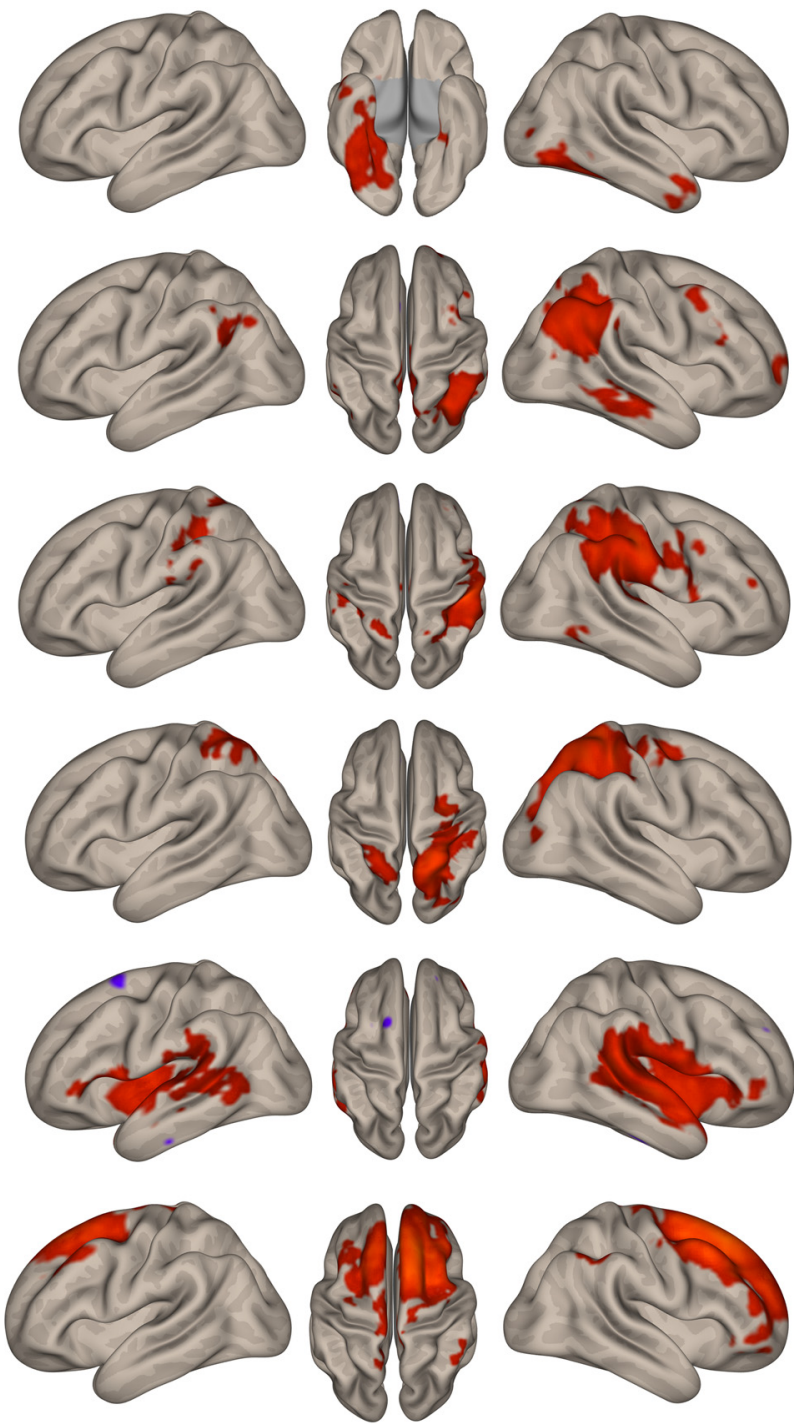
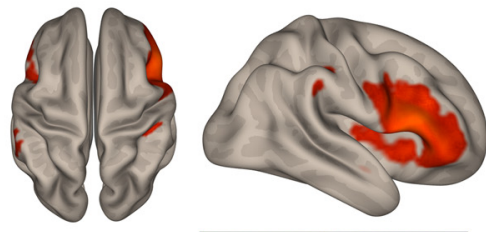

$t_{27}=-10.75$

Figure 2. Connectivity of each seed-ROI with the rest of the brain (left column, healthy controls; right column, stroke patients). Seed-ROls are as follows: (A) Fus, (B) Ang, (C) SMG, (D) PSup, $(\boldsymbol{E})$ STG, $(\boldsymbol{F})$ FSup, and $(\boldsymbol{G})$ Flnf. Reddish tones represent positive correlations. Bluish tones represent negative correlations.

spheric FC. Figure 4 shows a color-rendered matrix of mean connectivity for each ROI pair. Connectivity in controls was highest between homotopic region pairs (mean: $0.71 \pm 0.1$ ), followed by right intrahemispheric (mean: $0.56 \pm 0.12$ ), left intrahemispheric (mean: $0.48 \pm 0.12$ ), and heterotopic FC (mean: $0.45 \pm 0.14$ ). In patients, homotopic FC was highest (mean: $0.54 \pm 0.22$ ), followed by left intrahemispheric (mean: $0.49 \pm 0.17$ ), right intrahemispheric (mean: $0.42 \pm 0.16$ ), and heterotopic FC (mean: $0.28 \pm 0.18$ ). To examine these data statistically, we performed a mixed ANOVA with Group as between-factor and ROI-to-ROI condition as within-factor. This analysis yielded significant main effects of Group $\left(F_{(1,53)}=13.21, p=0.001, \eta^{2}=0.20\right)$ and Condition $\left(F_{(3,159)}\right.$ $\left.=51.53, p<0.0001, \eta^{2}=0.493\right)$, as well as a Group $\times$ Condition interaction $\left(F_{(3,159)}=8.41, p<0.0001, \eta^{2}=0.137\right)$. Independent- samples $t$ tests comparing groups across different conditions showed that, in all conditions, controls exhibited higher connectivity than patients $\left(t_{(53)}>3.76, p<0.001\right)$, except for left intrahemispheric FC $\left(t_{(53)}=0.23\right)$.

To summarize, the seed-based analyses revealed a consistent pattern of connectivity between each seed and neighboring regions of the same hemisphere, where distant within-hemisphere connections reflected known functional networks (in particular, frontoparietal networks identified in FC analyses with the Ang, PSup, FSup, and FInf seeds). ROI-to-ROI analyses showed that interhemispheric connections involving homotopic areas had the highest degree of efficiency in both groups. In addition, except for the intact left hemisphere, controls had consistently higher FC than patients. 
A
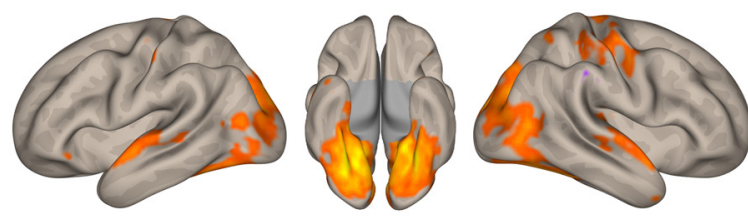

B
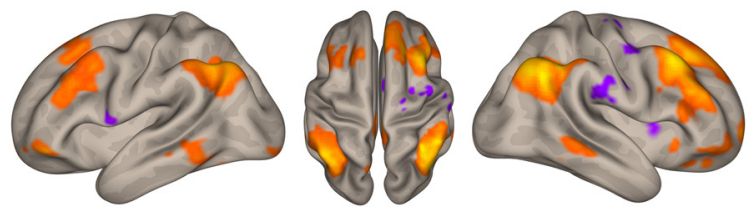

C
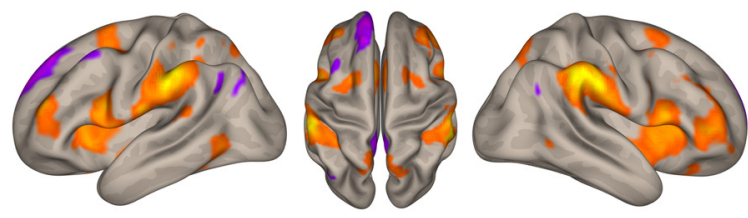

D
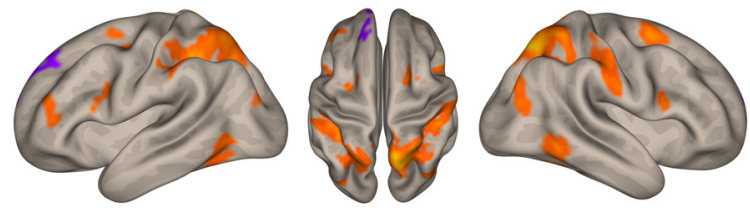

E
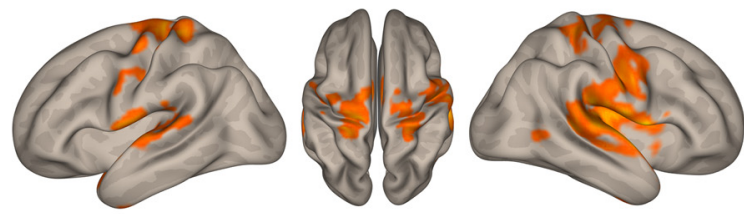

$\mathbf{F}$
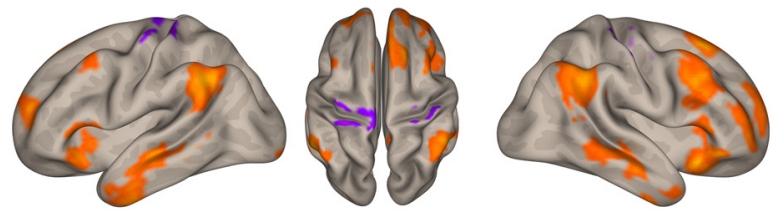

G
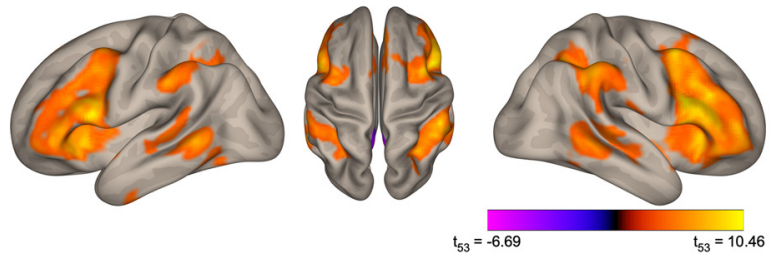

Figure 3. Direct contrast of seed-based connectivity between controls and stroke patients. Yellow-orange tones represent areas where controls had significantly higher correlations than patients. Pink represents areas of higher correlation in patients. Seed-ROls are as follows: (A) Fus, (B) Ang, (C) SMG, (D) PSup, (E) STG, ( $\boldsymbol{F})$ FSup, and (G) Finf.

\section{FC predictors of spatial cognition}

We next performed seed-based regression analyses that examined whether regional connectivity predicted behavioral scores in reading, line bisection, and cancellation. First, we examined FC predictors of reading performance. Unfortunately, reading errors were not linearly distributed across patients, as most patients made none or very few transformations characteristic of spatial dyslexia. Consequently, our cutoff identified only 7 patients with spatial dyslexia. Since these data did not lend themselves for regression analysis, we compared connectivity across groups with independent-samples $t$ tests. The main finding of these analyses was that patients with spatial dyslexia had increased FC between the right Fus and anterior inferior temporal cortex (cluster size: 192 voxels; MNI coordinates: $-60,-32$,
-18 ; FWE-corrected $p<0.02$; Fig. 5), but decreased FC between the left Fus and right posterior inferior temporo-occipital cortex (cluster size: 430 voxels; MNI coordinates: $-8,-64,-8$; FWEcorrected $p<0.0001$; Fig. $6 B$ ). In dyslexic patients, the left Fus seed additionally exhibited higher connectivity with the left insula (cluster size: 511 voxels; MNI coordinates: $-34,-10$, 4; FWE-corrected $p<0.0001$ ) and anterior temporal cortex (cluster size: 146 voxels; MNI coordinates: $-28,16,-46$; FWE-corrected $p<0.05)$. We repeated these analyses by including total lesion volume (instead of the relative volume of damage to the seedROI) as covariate and identified the same clusters of positive or negative connectivity. Thus, positive predictors of impaired reading were located in the left hemisphere, whereas negative predictors were all located in the right hemisphere.

We next searched for positive or negative predictors of the line bisection bias (where higher values indicate more severe deficit) by treating line bisection data as a continuous measure in a regression analysis. Of all right- and left-hemispheric seed-ROIs, only FC of the right Fus revealed clusters of significant FCbehavior relationship. In the larger of two clusters (located in the left superior occipital cortex), a significant decrease of connectivity was associated with greater ipsilesional bias on line bisection (Fig. 6 A; cluster size: 930 voxels; MNI coordinates: $-26,-86,28$; FWE-corrected $p<0.0001)$. Figure $6 B$ shows the negative relationship between mean cluster-FC and line bisection performance. These data followed a linear trend, which was very similar if the whole dataset was considered $\left(R^{2}=0.343\right)$, or only values of patients with pathologic scores $(>2$ SDs of the control mean; $R^{2}=0.308$ ). In a smaller cluster, located in the right occipital pole, increased connectivity predicted worse performance on the line bisection task (cluster size: 188 voxels; MNI coordinates: 18, $-104,-10$; FWE-corrected $p<0.05)$. Repeating these analyses with total lesion volume as covariate confirmed the same FCclusters without identifying any further significant results. We additionally performed group-based analyses by comparing 9 patients with a pathologic bias on line bisection to 19 unimpaired patients. Only FC of the right Fus identified a cluster of significant FC-behavior relationship, which was remarkably similar to the cluster identified with the regression analysis shown in Figure $6 A$ (cluster size: 884 voxels; MNI coordinates: $-28,-86$, 26; FWE-corrected $p<0.0001$ ). Thus, regression and groupbased analyses yielded very similar results, showing involvement of only one ROI (Fus) and identifying a cluster in the left superior occipital cortex in which FC predicted bias on line bisection.

Next, we examined predictors of left omissions in the Bells test, a sensitive measure of visual search deficits characterizing spatial neglect. Regression analyses yielded two clusters in which FC was negatively correlated with increasing numbers of omissions: one cluster was identified through the right SMG seed and was located in the left SMG (Fig. 7A; cluster size: 185 voxels; MNI coordinates: $-54,-30,18$; FWE-corrected $p<0.02$ ). A linear fit of these data was significant and very similar if computed across the whole dataset $\left(R^{2}=0.568\right)$, or only with the pathologic data ( $>3$ omissions; $\left.R^{2}=0.574\right)$. Analysis of left SMG FC revealed a second cluster located in the right insula (Fig. $7 B$; cluster size: 634 voxels; MNI coordinates: 50, 4, -10 ; FWE-corrected $p<0.0001)$. Again, the linear fit of these data was similar whether it was computed across the entire dataset $\left(R^{2}=0.718\right)$ or with pathologic scores only $\left(R^{2}=0.757\right)$. These two clusters were confirmed after repeating these analyses by including total lesion volume rather than the degree of damage to seed-ROIs as covariate. We also performed a group-based analysis on patients separated into an impaired $(N=13)$ and a nonimpaired group 
A Interhemispheric FC

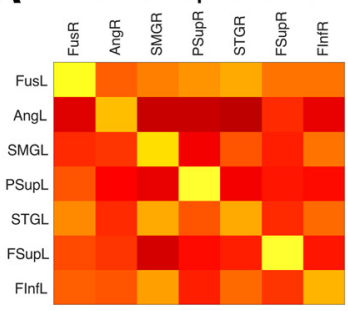

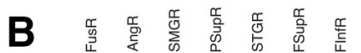

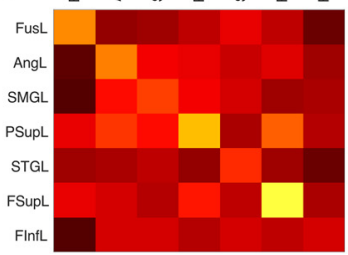

Intrahemispheric FC (LH)
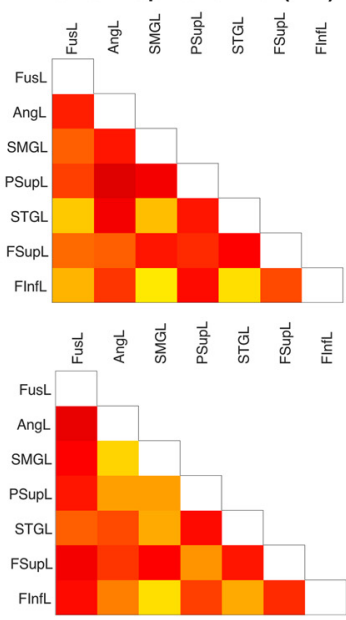

Intrahemispheric FC $(\mathrm{RH})$
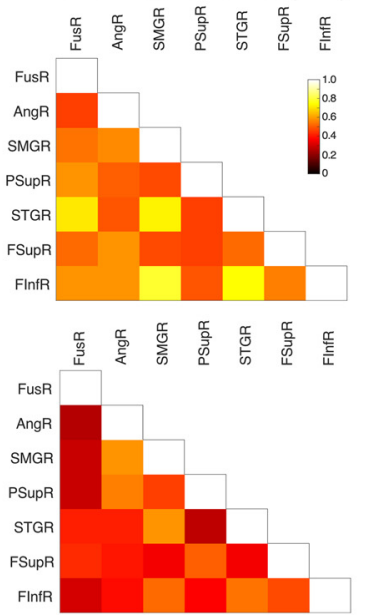

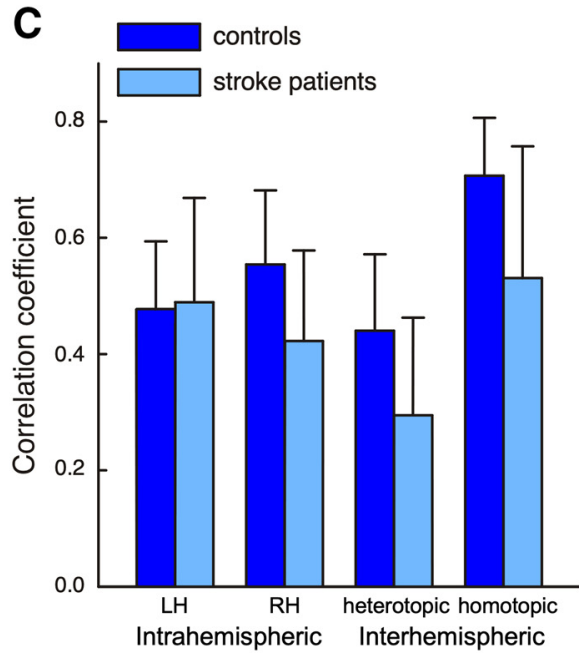

Figure 4. ROl-to-ROI-based analyses of interhemispheric and intrahemispheric connectivity. $\boldsymbol{A}$, Data of healthy controls. $\boldsymbol{B}$, Data of stroke patients. The colored matrices represent average interhemispheric FC for homotopic ROIs (shown on the descending diagonal axis) and heterotopic ROls, as well as intrahemispheric FC of the left hemisphere (LH) and right hemisphere (RH). $\boldsymbol{C}$, Average connectivity of controls and stroke patients for intrahemispheric and interhemispheric ROI pairs.
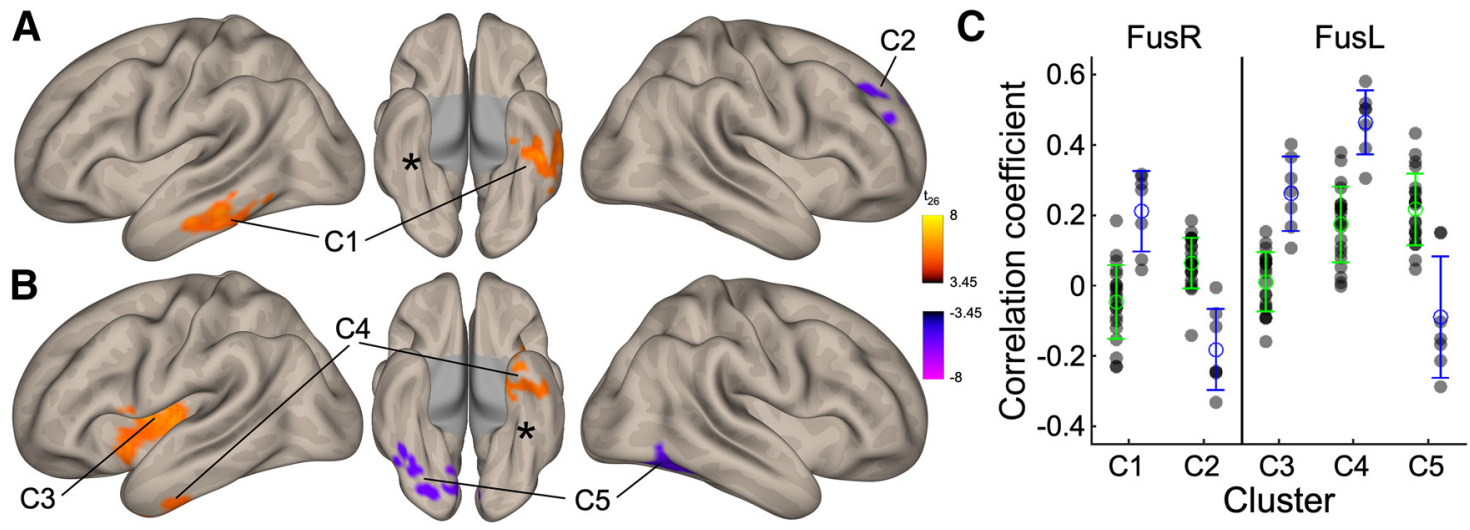

Figure 5. Seed-based analysis of $\mathrm{FC}$ as predictor of spatial dyslexia. $\boldsymbol{A}$, Clusters identified with the right Fus ROI $\left({ }^{*}\right)$. B, Clusters identified with the left Fus ROI. C, Average correlation values in each of the five clusters shown in $\boldsymbol{A}$ and $\boldsymbol{B}$. Green represents patients with spatial dyslexia. Blue represents nondyslexic patients.
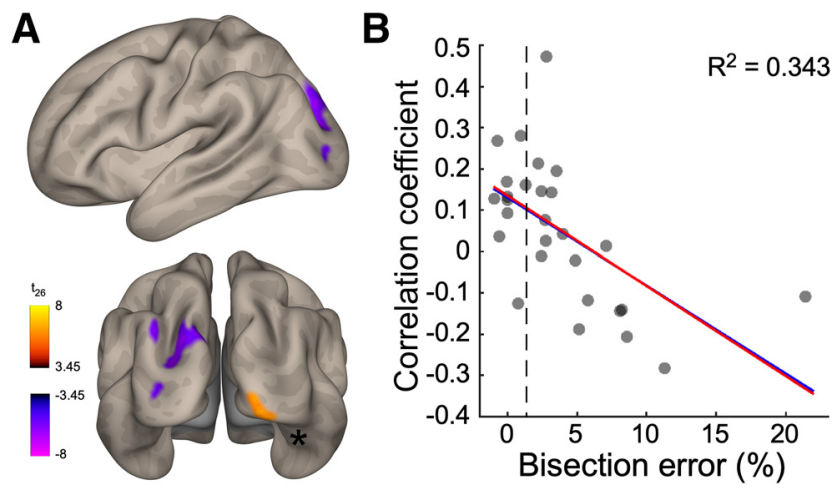

Figure 6. Seed-based analysis of $\mathrm{FC}$ as predictor of line bisection performance. $\boldsymbol{A}$, Clusters that significantly correlated with the right Fus ROI (*). B, Linear fit of bisection error and mean correlation within the cluster of negative correlation shown in $\boldsymbol{A}$. Stippled vertical line indicates the cutoff of normal performance. Blue line indicates fit for the whole dataset. Red line indicates only pathologic data.

$(N=15)$ by computing independent-samples $t$ tests. Of all seven ROIs, only FC of the left SMG revealed a cluster with a significant FC-behavior relationship. This cluster was similar to the cluster identified by the regression analysis and shown in Figure
$7 B$, although smaller (cluster size: 290 voxels; MNI coordinates: $36,14,-8$; FWE-corrected $p<0.0011$ ). Thus, regression and group-based analyses confirmed that connectivity between the left SMG and right insular cortex is a significant predictor of cancellation performance.

We finally explored the FC-behavior relationship between left- and right-hemisphere seeds by focusing on ROI-to-ROI interactions. For this analysis, we first extracted average correlations between interhemispheric and intrahemispheric ROI pairs and then correlated these with behavioral scores (line bisection bias and left omissions in the cancellation test). Figure $8 \mathrm{~A}$ illustrates color-rendered correlation matrices between connectivity and bias in line bisection, and Figure $8 B$ shows the correlation matrices for the number of omissions in the cancellation test. We performed one-way ANOVAs to compare FC between homotopic, heterotopic, left intrahemispheric, and right intrahemispheric ROI pairs. A significant effect $\left(F_{(3,90)}=10.39\right.$, $p<0.0001)$ indicated that heterotopic $(-0.22 \pm 0.16 ; p<0.003)$ and homotopic interhemispheric FC $(-0.34 \pm 0.14 ; p<0.001)$ were better (though negative) predictors of line bisection bias than left $(-0.07 \pm 0.13)$ and right intrahemispheric FC $(-0.07 \pm 0.16)$. The analysis of the cancellation data was also significant $\left(F_{(3,90)}=14.79, p<0.001\right)$; right intrahemispheric FC 
A
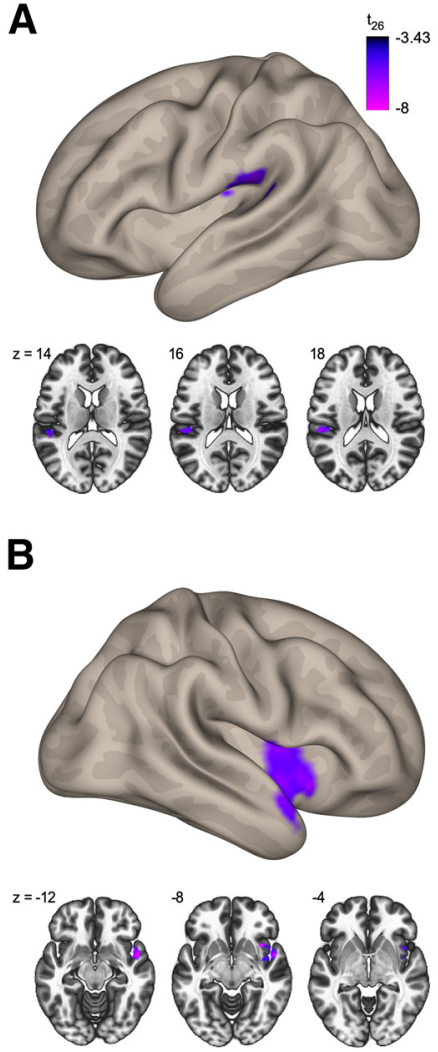

Figure 7. Seed-based analysis of cancellation performance. $\boldsymbol{A}$, Cluster functionally connected with the right SMG seed that negatively predicted the number of missed cancellation targets. $\boldsymbol{B}$, Cluster connected with the left SMG seed that negatively predicted the number of missed cancellation targets. Scatterplots represent individual connectivity. Stippled vertical line indicates the cutoff of normal performance. Blue line indicates fit for the whole dataset. Red line indicates only pathologic data.
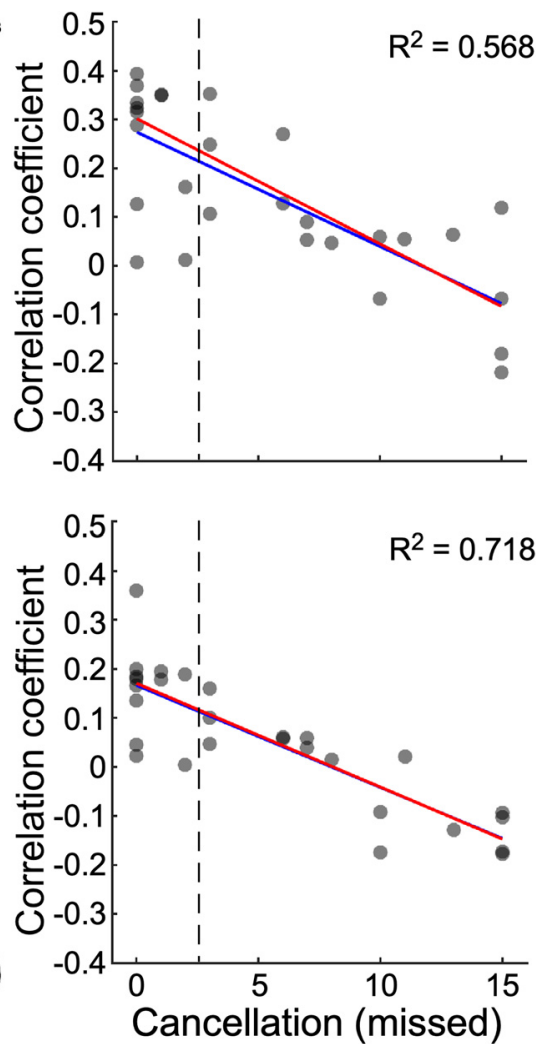

$(-0.22 \pm 0.14)$ and interhemispheric FC (heterotopic: $-0.16 \pm$ 0.16 ; homotopic: $-0.19 \pm 0.14$ ) were better (negative) predictors of cancellation bias than left intrahemispheric FC $(0.6 \pm 0.14$; all $p<0.001)$. Thus, although FC-behavior relationships based on ROI-to-ROI analysis were relatively weak in terms of the amplitude of correlation, a general pattern was that interhemispheric FC was a better predictor of behavioral deficits than left intrahemispheric connectivity.

\section{Discussion}

The present data show that behavioral scores in clinical tests commonly administered to assess deficits of spatial cognition are associated with distinct patterns of FC across the hemispheres. Seed-based analyses identified functional networks in healthy individuals that involved frontoparietal (Ang, SMarg, PSup, FSup, and FInf seed), occipito-temporal (Fus seed), and temporo-insular cortex (STG seed). These networks were well segregated and exhibited a high degree of bilateral symmetry, although the seed-ROIs were located in the right hemisphere. In contrast, patients showed intrahemispheric connectivity patterns that were largely limited to regions surrounding the seed. In addition, whereas controls showed a widespread connection pattern comprising homotopic and heterotopic regions of the contralateral hemisphere, interhemispheric connectivity in patients was restricted to homotopic cortex. This finding suggests that areas of the injured hemisphere maintain collaboration with homotopic areas but fail to connect to more distant and heterotopic regions located in the contralateral hemisphere. Indeed, ROI-to-ROI analyses of our patient sample showed that intrinsic connectivity of the damaged right hemisphere and between hemispheres was significantly decreased, whereas intrahemispheric FC of the left hemisphere was comparable to healthy controls. Disruption of connectivity with distant brain regions is in agreement with previous modeling (Alstott et al., 2009) and empirical studies (Carter et al., 2010; Gratton et al., 2012), suggesting a particular vulnerability of functional interactions which are conveyed by indirect structural connections (van den Heuvel and Hulshoff Pol, 2010; Hermundstad et al., 2013). Local cortical or subcortical damage may thus have widespread consequences on functional interactions between relatively distant brain regions. This principle provides a possible solution to controversies surrounding the anatomy of behavioral symptoms after stroke, in particular the contribution of preserved right-hemispheric cortex and areas of the undamaged left hemisphere, to spatial dyslexia or visual search deficits.

Our observation that cross-hemisphere changes of FC predict reading deficits is of direct relevance to the possible contribution of dorsal and ventral pathways to spatial reference frames used in reading. Spatial dyslexia is a reading disorder that is characterized by perceptual or attentional impairments, whereas phonological and semantic processes are intact (Ellis et al., 1987; Vallar et al., 2010). The defining feature of spatial dyslexia is an object-centered deficit (i.e., reading errors are determined by a reference frame centered on the word) that gives rise to lateralized omissions and substitutions (Hillis and Caramazza, 1995). Some previous lesion studies have shown that such object-centered failures reflect damage to superior or inferior parietal cortex (Ptak et al., 2012; Pedrazzini et al., 2017), whereas others pointed to more ventral regions, such as the lateral occipital or inferior temporal cortex (Lee et al., 2009; Medina et al., 2009). The present study indicates that, even if the Fus is nearly unaffected by stroke (only $\sim 6 \%$ of the Fus-ROI was damaged in the present study), its connectivity with the language-dominant left hemisphere is determinant for the occurrence of spatial dyslexia. We observed two patterns of FC changes that appear to be critical for spatial reading deficits. On the one hand, we found decreased connectivity between right inferior occipitotemporal cortex and the left Fus, a region known to play a central role in peripheral reading processes. Damage to the left Fus results in pure alexia, a disorder whose main characteristic is a letter-by-letter reading strategy (Gaillard et al., 2006; Pflugshaupt et al., 2009; Di Pietro et al., 2012). Decreased FC between left fusiform and right occipitotemporal cortex may therefore reflect the functional isolation of the right hemisphere from lefthemisphere areas that are essential for the visual and phonological aspects of reading. On the other hand, patients with spatial dyslexia had increased connectivity between the left and right Fus and more anterior temporal and insular cortex of the language-dominant hemisphere. This might be a 
A Interhemispheric FC

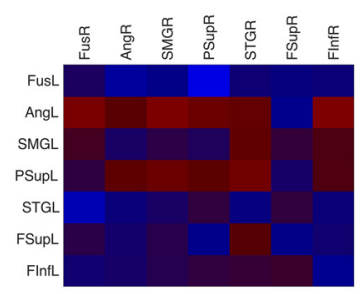

B

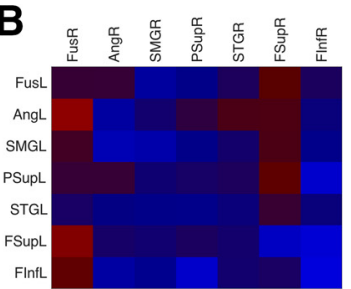

Intrahemispheric FC (LH)
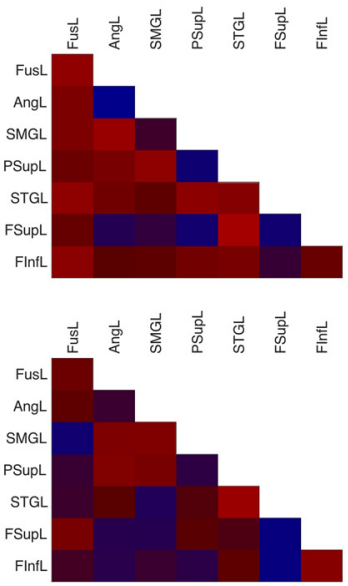

Intrahemispheric FC $(\mathrm{RH})$
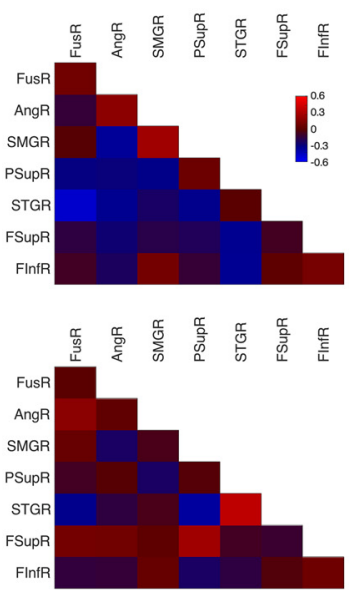

C

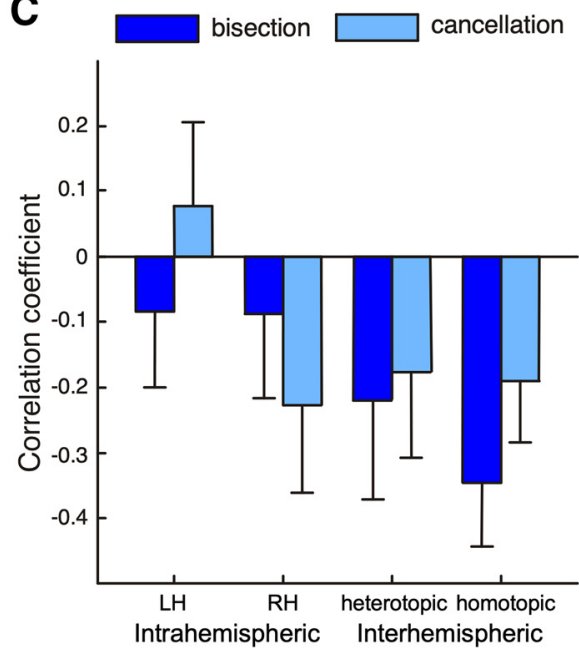

Figure 8. ROl-to-ROl-based analyses of connectivity-behavior relationships for homotopic, heterotopic, and intrahemispheric region pairs. $A$, Correlation between FC and the line bisection task. $\boldsymbol{B}$, Correlation between $\mathrm{FC}$ and the number of missed left cancellation targets. $C$, Average connectivity in the line bisection and cancellation tasks for intrahemispheric and interhemispheric ROl pairs.

compensatory adaptation, possibly reflecting increased recruitment of areas necessary for the interpretation of the degraded textual information.

In contrast to the FC increases observed in the reading task, the connectivity analyses of line bisection and cancellation revealed a negative association between connectivity and degree of deficit. Thus, negative FC between the right Fus and the left superior occipital cortex predicted line bisection bias. Several previous studies have observed a positive association between line bisection and reading, suggesting that both tasks may share common object-centered reference frames (Azouvi et al., 2002; Lee et al., 2009; Pedrazzini et al., 2017). This association might particularly engage the right Fus, which emerged as significant seed in the present study, both for reading and line bisection. However, while a connection between the right and left inferior temporal cortex was of significance for reading, FC with dorsal occipital cortex is more compatible with recruitment of processes necessary for stimulus-based spatial judgment in the line bisection task (Fink et al., 1997; Rorden et al., 2006). Thus, although the right Fus might mediate object-centered processing for reading and line bisection, its connections with distinct left-hemispheric regions differentiate between the two tasks.

Contrasting with the two stimulus-centered tasks, FC predictors of cancellation involved the right and left SMG, a region lying at the right temporoparietal junction that was previously identified by anatomic studies as crucial for the occurrence of spatial exploration deficits (Golay et al., 2008; Chechlacz et al., 2012; Molenberghs et al., 2012; Pedrazzini and Ptak, 2020). These analyses identified two interesting interactions: impaired performance was associated with decreased FC between the right SMG and its left homotopic region as well as decreased FC between the left SMG and the right insula. A decrease of interhemispheric FC between both SMGs has been observed previously (He et al., 2007) and is in line with the classic proposal that spatial neglect reflects a lack of balance between attention resources of both hemispheres. This hypothesis is based on the assumption that awareness is biased rightward in neglect because of the stronger allocation of attention to right space by the left hemisphere than to left space by the right hemisphere (Kinsbourne, 1993; Koch et al., 2008, 2011; Szczepanski et al., 2010). However, while previous studies related this attentional imbalance to impaired interhemispheric FC between superior parietal cortices (He et al., 2007; Baldassarre et al., 2014; Ramsey et al., 2016), none of our regression or group-based analyses implicated specifically this region. Our patient group as a whole showed decreased interhemispheric FC between all ROIs, not only the superior parietal cortex. One possible reason for this discrepancy is our focus on postacute patients, who exhibit less specific alterations of FC between dorsal parietal regions than patients in early phases of disease (Corbetta et al., 2005). Alternatively, the differences in findings may reflect different definitions of neglect: as a clinical syndrome defined by combined deficits in visual search, spatial extinction, and activities of daily life (Corbetta et al., 2005), or as independent cutoff performance in reading, line bisection, or cancellation. Since these different spatial tasks engage discrete cognitive processes, neglect defined in the latter way may reflect engagement of distinct brain regions.

Studying FC-behavior relationships in stroke patients may provide complementary information that helps to confirm or clarify some findings of previous VLSM or DTI studies. One such finding is our observation of decreased connectivity between the left SMG and the right insula, a region whose contribution to spatial attention and stimulus selection is currently under debate. Some authors have proposed that this region acts as a "switch" that biases or interrupts ongoing cortical activity to redirect attention toward stimuli that threaten homeostasis (Menon and Uddin, 2010; Uddin, 2015). Although lesion evidence supports a specialization of the insula for bodily awareness rather than attention for external stimuli (Jones et al., 2010; Pedrazzini and Ptak, 2019), some studies noted that insular damage might contribute to spatial neglect (Golay et al., 2008; Molenberghs et al., 2012). The fact that the present study only found involvement of the right insula when cancellation performance was measured suggests a specific contribution of this region to spatial exploration, but not to object-based measures, such as reading or line bisection. Another important finding of previous studies is the importance of transcallosal connections for the recovery of spatial neglect (Lunven et al., 2015) and the effectiveness of inhibitory contralesional stimulation to alleviate this disorder (Nyffeler et al., 2019). These observations are complemented by our finding that greater impairment in line 
bisection and cancellation is associated with decreased crosshemispheric FC, supporting the importance of interhemispheric cooperation in spatial cognition tasks.

In conclusion, our study indicates that cortical regions well outside the area of damage may contribute to deficits of spatial cognition. FC predictors were specific for each of the three tasks examined (reading, line bisection, and cancellation), supporting the assumption that interregional connectivity reflects distinct cognitive processes recruited during behavioral performance. These findings indicate that, similarly to direct tissue damage, functional adaptations distant from the lesion site are important predictors of deficit after stroke. Thus, the study of functional networks complements lesion mapping and DTI in the effort to acquire a deeper understanding of impaired spatial cognition after focal brain injury.

\section{References}

Alstott J, Breakspear M, Hagmann P, Cammoun L, Sporns O (2009) Modeling the impact of lesions in the human brain. PLoS Comput Biol 5: e1000408.

Amemiya S, Kunimatsu A, Saito N, Ohtomo K (2012) Impaired hemodynamic response in the ischemic brain assessed with BOLD fMRI. Neuroimage 61:579-590.

Azouvi P, Samuel C, Louis-Dreyfus A, Bernati T, Bartolomeo P, Beis JM, Chokron S, Leclercq M, Marchal F, Martin Y, de Montety G, Olivier S, Perennou D, Pradat-Diehl P, Prairial C, Rode G, Siéroff E, Wiart L, Rousseaux M, French Collaborative Study Group on Assessment of Unilateral Neglect (GEREN/GRECO) (2002) Sensitivity of clinical and behavioural tests of spatial neglect after right hemisphere stroke. J Neurol Neurosurg Psychiatry 73:160-166.

Baas U, de Haan B, Grassli T, Karnath HO, Mueri R, Perrig WJ, Wurtz P, Gutbrod K (2011) Personal neglect: a disorder of body representation? Neuropsychologia 49:898-905.

Baldassarre A, Ramsey L, Hacker CL, Callejas A, Astafiev SV, Metcalf NV, Zinn K, Rengachary J, Snyder AZ, Carter AR, Shulman GL, Corbetta M (2014) Large-scale changes in network interactions as a physiological signature of spatial neglect. Brain 137:3267-3283.

Bassett DS, Sporns O (2017) Network neuroscience. Nat Neurosci 20:353364.

Buckner RL, Krienen FM, Yeo BT (2013) Opportunities and limitations of intrinsic functional connectivity MRI. Nat Neurosci 16:832-837.

Buckner RL, Sepulcre J, Talukdar T, Krienen FM, Liu H, Hedden T, Andrews-Hanna JR, Sperling RA, Johnson KA (2009) Cortical hubs revealed by intrinsic functional connectivity: mapping, assessment of stability, and relation to Alzheimer's disease. J Neurosci 29:1860-1873.

Carter AR, Astafiev SV, Lang CE, Connor LT, Rengachary J, Strube MJ, Pope DL, Shulman GL, Corbetta M (2010) Resting interhemispheric functional magnetic resonance imaging connectivity predicts performance after stroke. Ann Neurol 67:365-375.

Chechlacz M, Rotshtein P, Humphreys GW (2012) Neuroanatomical dissections of unilateral visual neglect symptoms: ALE meta analysis of lesionsymptom mapping. Front Hum Neurosci 6:230.

Cole MW, Reynolds JR, Power JD, Repovs G, Anticevic A, Braver TS (2013) Multi-task connectivity reveals flexible hubs for adaptive task control. Nat Neurosci 16:1348-1355.

Committeri G, Pitzalis S, Galati G, Patria F, Pelle G, Sabatini U, CastriotaScanderbeg A, Piccardi L, Guariglia C, Pizzamiglio L (2007) Neural bases of personal and extrapersonal neglect in humans. Brain 130:431-441.

Corbetta M, Shulman GL (2011) Spatial neglect and attention networks. Annu Rev Neurosci 34:569-599.

Corbetta M, Kincade MJ, Lewis C, Snyder AZ, Sapir A (2005) Neural basis and recovery of spatial attention deficits in spatial neglect. Nat Neurosci 8:1603-1610.

Di Pietro M, Ptak R, Schnider A (2012) Preserved visual language identification despite severe alexia. Neuropsychologia 50:1327-1334.

Doricchi F, Tomaiuolo F (2003) The anatomy of neglect without hemianopia: a key role for parietal-frontal disconnection? Neuroreport 14:2239-2243.
Ellis AW, Flude BM, Young AW (1987) Neglect dyslexia and the early visual processing of letters in words and nonwords. Cogn Neuropsychol 4:439464.

Fellrath J, Mottaz A, Schnider A, Guggisberg AG, Ptak R (2016) Theta-band functional connectivity in the dorsal fronto-parietal network predicts goal-directed attention. Neuropsychologia 92:20-30.

Fink GR, Dolan RJ, Halligan PW, Marshall JC, Frith CD (1997) Space-based and object-based visual attention: shared and specific neural domains. Brain 120:2013-2028.

Gaillard R, Naccache L, Pinel P, Clemenceau S, Volle E, Hasboun D, Dupont S, Baulac M, Dehaene S, Adam C, Cohen L (2006) Direct intracranial, FMRI, and lesion evidence for the causal role of left inferotemporal cortex in reading. Neuron 50:191-204.

Gauthier L, Dehaut F, Joanette Y (1989) The Bells Test: a quantitative and qualitative test for visual neglect. Int J Clin Neuropsychol 11:49-54.

Golay L, Schnider A, Ptak R (2008) Cortical and subcortical anatomy of chronic spatial neglect following vascular damage. Behav Brain Funct $4: 43$.

Goveas JS, Xie C, Chen G, Li W, Ward BD, Franczak MB, Jones JL, Antuono PG, Li SJ (2013) Functional network endophenotypes unravel the effects of apolipoprotein E epsilon 4 in middle-aged adults. PLoS One 8:e55902.

Gratton C, Nomura EM, Perez F, D'Esposito M (2012) Focal brain lesions to critical locations cause widespread disruption of the modular organization of the brain. J Cogn Neurosci 24:1275-1285.

He BJ, Snyder AZ, Vincent JL, Epstein A, Shulman GL, Corbetta M (2007) Breakdown of functional connectivity in frontoparietal networks underlies behavioral deficits in spatial neglect. Neuron 53:905-918.

Hermundstad AM, Bassett DS, Brown KS, Aminoff EM, Clewett D, Freeman S, Frithsen A, Johnson A, Tipper CM, Miller MB, Grafton ST, Carlson JM (2013) Structural foundations of resting-state and task-based functional connectivity in the human brain. Proc Natl Acad Sci USA 110:6169-6174.

Hillis AE, Caramazza A (1995) A framework for interpreting distinct patterns of hemispatial neglect. Neurocase 1:189-207.

Jones CL, Ward J, Critchley HD (2010) The neuropsychological impact of insular cortex lesions. J Neurol Neurosurg Psychiatry 81:611-618.

Karnath HO, Fruhmann Berger M, Küker W, Rorden C (2004) The anatomy of spatial neglect based on voxelwise statistical analysis: a study of 140 patients. Cereb Cortex 14:1164-1172.

Kinsbourne M (1993) Orientational bias model of unilateral neglect: evidence from attentional gradients within hemispace. In: Unilateral neglect: clinical and experimental studies (Robertson IH, Marshall JC, eds), pp 63-86. Hove, UK: Lawrence Erlbaum.

Koch G, Cercignani M, Bonni S, Giacobbe V, Bucchi G, Versace V, Caltagirone C, Bozzali M (2011) Asymmetry of parietal interhemispheric connections in humans. J Neurosci 31:8967-8975.

Koch G, Oliveri M, Cheeran B, Ruge D, Lo Gerfo E, Salerno S, Torriero S, Marconi B, Mori F, Driver J, Rothwell JC, Caltagirone C (2008) Hyperexcitability of parietal-motor functional connections in the intact left-hemisphere of patients with neglect. Brain 131:3147-3155.

Lee BH, Suh MK, Kim EJ, Seo SW, Choi KM, Kim GM, Chung CS, Heilman KM, Na DL (2009) Neglect dyslexia: frequency, association with other hemispatial neglects, and lesion localization. Neuropsychologia 47:704710.

Lunven M, Thiebaut De Schotten M, Bourlon C, Duret C, Migliaccio R, Rode G, Bartolomeo P (2015) White matter lesional predictors of chronic visual neglect: a longitudinal study. Brain 138:746-760.

Lv Y, Margulies DS, Cameron Craddock R, Long X, Winter B, Gierhake D, Endres M, Villringer K, Fiebach J, Villringer A (2013) Identifying the perfusion deficit in acute stroke with resting-state functional magnetic resonance imaging. Ann Neurol 73:136-140.

Medina J, Kannan V, Pawlak MA, Kleinman JT, Newhart M, Davis C, Heidler-Gary JE, Herskovits EH, Hillis AE (2009) Neural substrates of visuospatial processing in distinct reference frames: evidence from unilateral spatial neglect. J Cogn Neurosci 21:2073-2084.

Menon V, Uddin LQ (2010) Saliency, switching, attention and control: a network model of insula function. Brain Struct Funct 214:655-667.

Molenberghs P, Gillebert CR, Peeters R, Vandenberghe R (2008) Convergence between lesion-symptom mapping and functional magnetic resonance imaging of spatially selective attention in the intact brain. J Neurosci 28:3359-3373. 
Molenberghs P, Sale MV, Mattingley JB (2012) Is there a critical lesion site for unilateral spatial neglect? A meta analysis using activation likelihood estimation. Front Hum Neurosci 6:78.

Mort DJ, Malhotra P, Mannan SK, Rorden C, Pambakian A, Kennard C, Husain M (2003) The anatomy of visual neglect. Brain 126:1986-1997.

Nachev P, Coulthard E, Jager HR, Kennard C, Husain M (2008) Enantiomorphic normalization of focally lesioned brains. Neuroimage 39:1215-1226.

Nyffeler T, Vanbellingen T, Kaufmann BC, Pflugshaupt T, Bauer D, Frey J, Chechlacz M, Bohlhalter S, Muri RM, Nef T, Cazzoli D (2019) Theta burst stimulation in neglect after stroke: functional outcome and response variability origins. Brain 142:992-1008.

Pedrazzini E, Ptak R (2019) Damage to the right temporoparietal junction, but not lateral prefrontal or insular cortex, amplifies the role of goaldirected attention. Sci Rep 9:306.

Pedrazzini E, Ptak R (2020) The neuroanatomy of spatial awareness: a largescale region-of-interest and voxel-based anatomical study. Brain Imaging Behav 14:615-626.

Pedrazzini E, Schnider A, Ptak R (2017) A neuroanatomical model of spacebased and object-centered processing in spatial neglect. Brain Struct Funct 222:3605-3613.

Petersen SE, Sporns O (2015) Brain networks and cognitive architectures. Neuron 88:207-219.

Pflugshaupt T, Gutbrod K, Wurtz P, von Wartburg R, Nyffeler T, de Haan B, Karnath HO, Mueri RM (2009) About the role of visual field defects in pure alexia. Brain 132:1907-1917.

Pineiro R, Pendlebury S, Johansen-Berg H, Matthews PM (2002) Altered hemodynamic responses in patients after subcortical stroke measured by functional MRI. Stroke 33:103-109.

Poldrack RA, Farah MJ (2015) Progress and challenges in probing the human brain. Nature 526:371-379.

Power JD, Barnes KA, Snyder AZ, Schlaggar BL, Petersen SE (2012) Spurious but systematic correlations in functional connectivity MRI networks arise from subject motion. Neuroimage 59:2142-2154.

Price CJ, Friston KJ (2002) Degeneracy and cognitive anatomy. Trends Cogn Sci 6:416-421.

Ptak R, Schnider A, Golay L, Müri R (2007) A non-spatial bias favouring fixated stimuli revealed in patients with spatial neglect. Brain 130:32113222.

Ptak R, Di Pietro M, Schnider A (2012) The neural correlates of object-centered processing in reading: a lesion study of neglect dyslexia. Neuropsychologia 50:1142-1150.

Ptak R, Schnider A, Fellrath J (2017) The dorsal frontoparietal network: a core system for emulated action. Trends Cogn Sci 21:589-599.

Ramsey LE, Siegel JS, Baldassarre A, Metcalf NV, Zinn K, Shulman GL, Corbetta M (2016) Normalization of network connectivity in hemispatial neglect recovery. Ann Neurol 80:127-141.

Ronchi R, Algeri L, Chiapella L, Spada S, Vallar G (2012) Spatial neglect and perseveration in visuomotor exploration. Neuropsychology 26:588-603.

Rorden C, Bonilha L, Fridriksson J, Bender B, Karnath HO (2012) Age-specific CT and MRI templates for spatial normalization. Neuroimage 61:957-965.

Rorden C, Fruhmann Berger M, Karnath HO (2006) Disturbed line bisection is associated with posterior brain lesions. Brain Res 1080:17-25.

Rorden C, Karnath HO, Bonilha L (2007) Improving lesion-symptom mapping. J Cogn Neurosci 19:1081-1088.
Rossini PM, Altamura C, Ferretti A, Vernieri F, Zappasodi F, Caulo M, Pizzella V, Del Gratta C, Romani GL, Tecchio F (2004) Does cerebrovascular disease affect the coupling between neuronal activity and local haemodynamics? Brain 127:99-110.

Shallice T, Cooper RP (2011) The organisation of mind. Oxford: Oxford UP.

Siegel JS, Ramsey LE, Snyder AZ, Metcalf NV, Chacko RV, Weinberger K, Baldassarre A, Hacker CD, Shulman GL, Corbetta M (2016) Disruptions of network connectivity predict impairment in multiple behavioral domains after stroke. Proc Natl Acad Sci USA 113:E4367-E4376.

Siegel JS, Shulman GL, Corbetta M (2017) Measuring functional connectivity in stroke: approaches and considerations. J Cereb Blood Flow Metab 37:2665-2678

Sperber C, Karnath HO (2018) On the validity of lesion-behaviour mapping methods. Neuropsychologia 115:17-24

Szczepanski SM, Konen CS, Kastner S (2010) Mechanisms of spatial attention control in frontal and parietal cortex. J Neurosci 30:148-160.

Tang C, Zhao Z, Chen C, Zheng X, Sun F, Zhang X, Tian J, Fan M, Wu Y, Jia $J$ (2016) Decreased functional connectivity of homotopic brain regions in chronic stroke patients: a resting state fMRI study. PLoS One 11: e0152875.

Thiebaut de Schotten M, Tomaiuolo F, Aiello M, Merola S, Silvetti M, Lecce F, Bartolomeo P, Doricchi F (2014) Damage to white matter pathways in subacute and chronic spatial neglect: a group study and 2 single-case studies with complete virtual "in vivo" tractography dissection. Cereb Cortex 24:691-706.

Toba MN, Zavaglia M, Rastelli F, Valabrégue R, Pradat-Diehl P, ValeroCabré A, Hilgetag CC (2017) Game theoretical mapping of causal interactions underlying visuo-spatial attention in the human brain based on stroke lesions. Hum Brain Mapp 38:3454-3471.

Tzourio-Mazoyer N, Landeau B, Papathanassiou D, Crivello F, Etard O, Delcroix N, Mazoyer B, Joliot M (2002) Automated anatomical labeling of activations in SPM using a macroscopic anatomical parcellation of the MNI MRI single-subject brain. Neuroimage 15:273-289.

Uddin LQ (2015) Salience processing and insular cortical function and dysfunction. Nat Rev Neurosci 16:55-61.

Urbanski M, Thiebaut de Schotten M, Rodrigo S, Oppenheim C, Touze E, Meder JF, Moreau K, Loeper-Jeny C, Dubois B, Bartolomeo P (2011) DTI-MR tractography of white matter damage in stroke patients with neglect. Exp Brain Res 208:491-505.

Vallar G, Perani D (1986) The anatomy of unilateral neglect after right-hemisphere stroke lesions: a clinical/CT scan correlation study in man. Neuropsychologia 24:609-622.

Vallar G, Burani C, Arduino LS (2010) Neglect dyslexia: a review of the neuropsychological literature. Exp Brain Res 206:219- 235.

van den Heuvel MP, Hulshoff Pol HE (2010) Exploring the brain network: a review on resting-state fMRI functional connectivity. Eur Neuropsychopharmacol 20:519-534.

Vandenberghe R, Molenberghs P, Gillebert CR (2012) Spatial attention deficits in humans: the critical role of superior compared to inferior parietal lesions. Neuropsychologia 50:1092-1103.

Whitfield-Gabrieli S, Nieto-Castanon A (2012) CONN: a functional connectivity toolbox for correlated and anticorrelated brain networks. Brain Connect 2:125-141.

Yeo BT, Krienen FM, Sepulcre J, Sabuncu MR, Lashkari D, Hollinshead M, Roffman JL, Smoller JW, Zollei L, Polimeni JR, Fischl B, Liu H, Buckner RL (2011) The organization of the human cerebral cortex estimated by intrinsic functional connectivity. J Neurophysiol 106:1125-1165. 